\title{
Heavy-lepton production via vector-boson fusion
}

\author{
O. J. P. Éboli \\ Departamento de Física, Universidade Estadual Paulista "Júlio de Mesquita Filho," IGCE, \\ C.P. 178, 13500, Rio Claro, São Paulo, Brazil \\ G. C. Marques and S. F. Novaes \\ Departamento de Física Matemática, Instituto de Física, Universidade de São Paulo, \\ C.P. 20516, 01498, São Paulo, Brazil \\ A. A. Natale \\ Instituto de Física Teórica, C.P. 5956, 01405, São Paulo, Brazil \\ (Received 20 March 1986)
}

\begin{abstract}
We investigate the production of a possible pair of sequential heavy charged leptons $\left(M_{L} \sim 200-800 \mathrm{GeV}\right)$ at $p p$ multi-TeV colliders via vector-boson fusion. The results are compared with the usual Drell-Yan mechanism and with the two-photon process. For very heavy leptons the fusion of $W^{\prime}$ s provides a contribution that is, at high energies, larger than the Drell-Yan mechanism.
\end{abstract}

\section{INTRODUCTION}

Within the current model of electroweak interaction there is no satisfactory explanation for the existence of several generations of quarks and leptons. On the other hand, there is nothing, in principle, forbidding the existence of at least one more family beyond the usual ones. Since the search for new heavy elementary particles will become an important issue with the advent of multi- $\mathrm{TeV}$ hadron colliders, it is interesting to investigate mechanisms that enable the production of such a new family.

In this paper we shall be concerned with the production of heavy charged leptons, belonging to a new generation of sequential leptons $\left(v_{L}, L\right)$ in the framework of the standard model of electroweak interaction. The charged lepton is assumed to be very heavy, and its neutral partner massless.

The approach used here for studying lepton pair production in $p p$ and $p \bar{p}$ collisions is reminiscent of the parton-model ideas in which the high-energy proton (or antiproton) is viewed as a collection of quasifree partons. For low energies the only relevant partons are the quarks and gluons, and one expects that the Drell-Yan mechanism gives the most relevant contribution. As we increase the energy, however, other particles in the parton sea might play an important role. The treatment of massive vector bosons as constituents of the proton is the basis of the so-called effective-vector-boson approximation. This approximation, as applied to the specific problem we are interested in, has been developed by Kane, Repko, and Rolnick, ${ }^{1}$ and Dawson. ${ }^{2}$

The question to which we address ourselves in this paper is the study of the various vector-boson fusion processes contributing to the production of a pair of charged leptons in $p p$ and $p \bar{p}$ collisions. Figure 1 exhibits a prototype of the reactions considered by us. In particular we will be concerned with the possibility that some of these processes dominate over the usual Drell-Yan mechanism $\left(q \bar{q} \rightarrow \gamma\right.$ or $\left.Z \rightarrow L^{+} L^{-}\right)$and the two-photon process $\left(\gamma \gamma \rightarrow L^{+} L^{-}\right)$at very high energies.

In the next section we introduce the basic expressions for the study of heavy-dilepton production, and the cross sections for the elementary processes relevant to our problem. Section III contains our results and discussions.

\section{VECTOR-BOSON FUSION MECHANISMS FOR MASSIVE-LEPTON-PAIR PRODUCTION}

In order to compute the contributions of the fusion of different vector partons to dilepton production, we shall use the effective-vector-boson approximation. A basic ingredient in all calculations, within this approximation, are the distribution functions. For massive vector particles, we treat separately the contributions due to transverse and longitudinal polarizations. ${ }^{1,2}$

The probability distribution of transverse vector bosons in a quark in the high-energy limit, i.e., when the quark

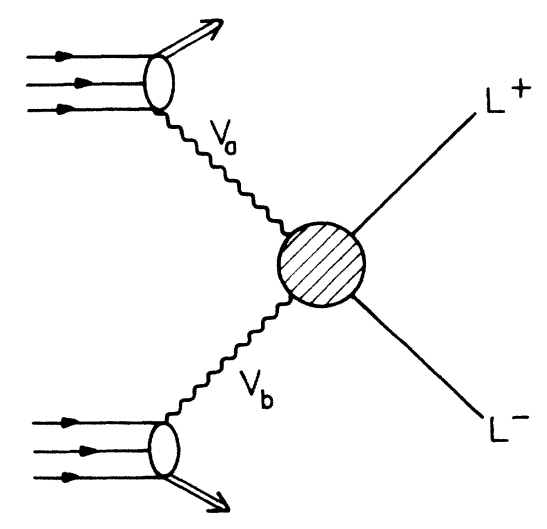

FIG. 1. Lepton pair production via vector-boson fusion. 
subenergy $E$ is much larger than the vector-boson mass $\left(M_{V}\right)$, is given by ${ }^{2}$

$$
V_{q}^{T}(x) \cong \frac{\left(C_{V}^{2}+C_{A}^{2}\right)}{8 \pi^{2}} \frac{\left[x^{2}+2(1-x)\right]}{x} \ln \left(\frac{4 E^{2}}{M_{V}^{2}}\right),
$$

whereas the probability distribution of longitudinal vector bosons in a quark, in the same limit, is

$$
V_{q}^{L}(x) \cong \frac{\left(C_{V}^{2}+C_{A}^{2}\right)}{4 \pi^{2}} \frac{(1-x)}{x},
$$

where

$$
C_{V}=\left(g / \cos \theta_{W}\right)\left(\frac{1}{2} T_{3 L}-Q \sin ^{2} \theta_{W}\right)
$$

and

$$
C_{A}=-\left(g / \cos \theta_{W}\right)\left(\frac{1}{2} T_{3 L}\right) \text { for } V=Z^{0} ;
$$

whereas $C_{V}=-C_{A}=g / 2 \sqrt{2}$ for $V=W^{ \pm} . T_{3 L}$ is the third component of the weak isospin and $Q$ is the quark electric charge.

The vector-boson distribution in a hadron is obtained by the convolution of (1) and (2) with the quark distribution functions in the hadron $q_{h}(x)$; that is

$$
V_{h}(x)=\sum_{i} \int_{x_{\min }}^{1} \frac{d y}{y} q_{h}^{i}(y) V_{q i}(x / y)
$$

where the sum is carried out over the quark and antiquark relevant flavors.

We are interested in computing the different vectorboson fusion contributions to the rapidity distribution at $y=0$ for the process $h_{1} h_{2} \rightarrow L^{+} L^{-}+X\left[h_{1,2}=p(\bar{p})\right]$. This quantity gives a good estimate of the total cross section, because the rapidity distribution is flat in the considered range. In terms of the distribution functions associated to the vector bosons, it can be written as

$$
\begin{aligned}
& \left.\frac{d \sigma^{h_{1} h_{2} \rightarrow L^{+}+L^{-}}}{d y}\right|_{y=0} \\
& =\int_{4 M_{L}{ }^{2} / s}^{1} d \tau\left[V_{h_{1}}^{a}(\sqrt{\tau}) V_{h_{2}}^{b}(\sqrt{\tau})\right. \\
& \quad+(a \leftrightarrow b)] \hat{\sigma}_{V_{a} V_{b} \rightarrow L^{+} L^{-}}(\tau s),
\end{aligned}
$$

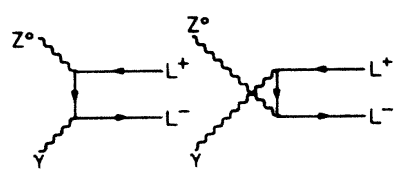

(a)

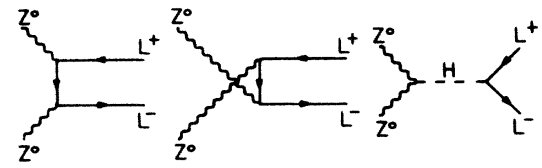

(b)

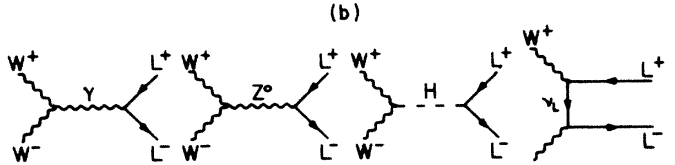

(c)

FIG. 2. Subprocesses that contribute to the heavy-lepton pair production: (a) $Z^{0} \gamma \rightarrow L^{+} L^{-}, \quad$ (b) $Z^{0} Z^{0} \rightarrow L^{+} L^{-}$, (c) $W^{+} W^{-} \rightarrow L^{+} L^{-}$.

where $\hat{\sigma}_{V_{a} V_{b} \rightarrow L^{+} L^{-}}(\tau s)$ is the cross section for the subprocesses of Fig. 2, $\sqrt{\hat{s}}$ is the total c.m. subprocess energy and $\tau=\hat{s} / s$.

Since we have already considered the distribution functions we now concentrate on the elementary processes of Fig. 2. We shall consider the production of a heavy charged lepton pair as a result of the subprocesses
(a) $Z^{0} \gamma \rightarrow L^{+} L^{-}$[Fig. 2(a)],
(b) $Z^{0} Z^{0} \rightarrow L^{+} L^{-}$[Fig. (2b)],
(c) $W^{+} W^{-} \rightarrow L^{+} L^{-}$[Fig. (2c)],

where the massive vector bosons are taken transversely and longitudinally polarized.

In the following sections we shall give the results of the cross sections for the subprocesses shown in Fig. 2, for massive vector bosons transversely and longitudinally polarized. In all calculations we have assumed $\hat{s} \gg M_{V}{ }^{2}$ and $M_{L}>M_{V}$.

\section{A. $Z^{0} \gamma$ fusion}

At first we consider the subprocess $Z^{0} \gamma \rightarrow L^{+} L^{-}$of Fig. 2(a). The cross section for the transversely polarized $Z^{0}$ is

$$
\hat{\sigma}\left(Z_{T}^{0} \gamma \rightarrow L^{+} L^{-}\right)=\frac{\pi \alpha^{2}}{4 \sin ^{2} \theta_{W} \cos ^{2} \theta_{W}} \frac{\beta}{\hat{s}}\left[\left(1-4 \sin ^{2} \theta_{W}\right)^{2}\left[-\beta^{2}+\frac{\left(1+\beta^{4}\right)}{2 \beta} \mathscr{L}\right]-1+3\left(1-\beta^{2}\right)+\frac{\left(3 \beta^{4}-1\right)}{2 \beta} \mathscr{L}\right],
$$

where

$$
\beta=\left(1-4 \frac{M_{L}^{2}}{\hat{s}}\right]^{1 / 2}
$$

is the lepton velocity in the c.m. frame, and

$$
\mathscr{L} \equiv \ln \frac{(1+\beta)}{(1-\beta)} .
$$


The cross section for the longitudinally polarized $Z^{0}$ is

$$
\left.\hat{\sigma}\left(Z_{L}^{0} \gamma \rightarrow L^{+} L^{-}\right)=\frac{\pi \alpha^{2}}{\sin ^{2} \theta_{W} \cos ^{2} \theta_{W}}\left(1-4 \sin ^{2} \theta_{W}\right)^{2} \frac{\beta}{\hat{s}} \frac{M_{L}^{2}}{M_{Z}^{2}} \mid-2+\frac{1}{\beta} \mathscr{L}\right) .
$$

\section{B. $Z^{0} Z^{0}$ fusion}

The diagrams contributing to $Z^{0} Z^{0}$ fusion are shown in Fig. 2(b), where $H$ is the standard Higgs boson. For transversely polarized $Z^{0}$ bosons the cross section is

$$
\begin{aligned}
\hat{\sigma}\left(Z_{T}^{0} Z_{T}^{0} \rightarrow L^{+} L^{-}\right)= & \frac{\pi \alpha^{2}}{64 \sin ^{4} \theta_{W} \cos ^{4} \theta_{W}} \frac{\beta}{\hat{s}} \\
\times & {\left[\left(1-4 \sin ^{2} \theta_{W}\right)^{4}\left(-1+3\left(1-\beta^{2}\right)+\frac{\left[2 \beta^{2}-\left(1-\beta^{2}\right)^{2}\right]}{2 \beta} \mathscr{L}\right)\right.} \\
& -2\left(1-4 \sin ^{2} \theta_{W}\right)^{2}\left(6+\beta^{2}-\frac{\left[6+\left(1-\beta^{2}\right)^{2}\right]}{2 \beta} \mathscr{L}+4 M_{L}{ }^{2} \beta \chi_{H}\left(\hat{s}-M_{H}{ }^{2}\right) \mathscr{L}\right] \\
& \left.-1-\left(1-\beta^{2}\right)+\frac{\left(3-\beta^{4}\right)}{2 \beta} \mathscr{L}+8 M_{L}{ }^{2} \beta \chi_{H}\left(\hat{s}-M_{H}{ }^{2}\right) \mathscr{L}+16 M_{L}{ }^{2} \beta^{2} \chi_{H} \hat{s}\right],
\end{aligned}
$$

where

$$
\chi_{H}=\frac{1}{\left(\hat{s}-M_{H}^{2}\right)^{2}+\Gamma_{H}^{2} M_{H}^{2}},
$$

$M_{H}$ and $\Gamma_{H}$ being the Higgs-boson mass and width, respectively.

When the $Z^{0}$ bosons are longitudinally polarized, we have

$$
\begin{aligned}
\hat{\sigma}\left(Z_{L}^{0} Z_{L}^{0} \rightarrow L^{+} L^{-}\right)= & \frac{\pi \alpha^{2}}{8 \sin ^{4} \theta_{W} \cos ^{4} \theta_{W}} \frac{M_{L}{ }^{2}}{M_{Z}{ }^{4}} \beta \\
\times & \quad\left(1-4 \sin ^{2} \theta_{W}\right)^{4}-2\left(1-4 \sin ^{2} \theta_{W}\right)^{2} \chi_{H} \hat{s}\left(\hat{s}-M_{H}{ }^{2}\right)+\beta^{2} \chi_{H} \hat{s}^{2} \\
& \left.\quad-\frac{\left(1-\beta^{2}\right)}{2 \beta}\left[\left(1-4 \sin ^{2} \theta_{W}\right)^{4}-2\left(1-4 \sin ^{2} \theta_{W}\right)^{2} \chi_{H} \hat{s}\left(\hat{s}-M_{H}{ }^{2}\right)\right] \mathscr{L}\right) .
\end{aligned}
$$

We do not worry about cross sections involving mixed polarizations $(T L)$, since $\sigma_{T T}$ and $\sigma_{L L}$ are enough to characterize the complete behavior of the process.

\section{C. $W^{+} W^{-}$fusion}

We shall consider here the possibility of the heavy lepton pair to be produced via the fusion of charged vector bosons [Fig. 2(c)]. For transversely polarized $W$ bosons the cross section is

$$
\hat{\sigma}\left(W_{T}^{+} W_{T}^{-} \rightarrow L^{+} L^{-}\right)=\sum_{i, j} \hat{\sigma}_{i j}^{T},
$$

where $\hat{\sigma}_{i j}(i, j=A, Z, H, v)$ are the contribution of each diagram of Fig. 2(c) and its relevant interferences. We get, for these contributions,

$$
\begin{aligned}
& \hat{\sigma}_{A A}^{T}=\frac{\pi \alpha^{2}}{3 \hat{s}} \beta\left(3-\beta^{2}\right), \\
& \hat{\sigma}_{Z Z}^{T}=\frac{\pi \alpha^{2}}{48 \sin ^{4} \theta_{W}} \frac{\beta}{\hat{s}}\left[\left(1-4 \sin ^{2} \theta_{W}\right)^{2}\left(3-\beta^{2}\right)+2 \beta^{2}\right], \\
& \hat{\sigma}_{H H}^{T}=\frac{\pi \alpha^{2}}{4 \sin ^{4} \theta_{W}} M_{L}^{2} \beta^{3} \chi_{H}, \\
& \left.\hat{\sigma}_{v v}^{T}=\frac{\pi \alpha^{2}}{8 \sin ^{4} \theta_{W}} \frac{\beta}{\hat{s}} \mid-\frac{2}{3}\left(3+\beta^{2}\right)+\left(1+\beta^{2}\right)\left(1-2 \beta^{2}\right)+\left[2\left(3+\beta^{2}\right)-\left(1-\beta^{4}\right)\left(1+2 \beta^{2}\right)\right] \frac{\mathscr{L}}{2 \beta}\right),
\end{aligned}
$$




$$
\begin{aligned}
& \hat{\sigma}_{A Z}^{T}=\frac{\pi \alpha^{2}}{6 \sin ^{2} \theta_{W}}\left(1-4 \sin ^{2} \theta_{W}\right) \frac{\beta}{\hat{s}}\left(3-\beta^{2}\right), \\
& \hat{\sigma}_{H v}^{T}=\frac{\pi \alpha^{2}}{4 \sin ^{4} \theta_{W}} M_{L}{ }^{2} \chi_{H}\left(\hat{s}-M_{H}{ }^{2}\right) \frac{\beta\left(1-\beta^{2}\right)}{\hat{S}}\left[-1+\frac{\left(1+\beta^{2}\right)}{2 \beta} \mathscr{L}\right], \\
& \hat{\sigma}_{A v}^{T}=\frac{-\pi \alpha^{2}}{8 \sin ^{2} \theta_{W}} \frac{\beta}{\hat{s}}\left[\frac{4}{3}\left(3-\beta^{2}\right)+\left(1-\beta^{4}\right)+\frac{\left(1-\beta^{2}\right)}{2 \beta}\left[3-\beta^{2}\left(2+\beta^{2}\right)\right] \mathscr{L}\right], \\
& \hat{\sigma}_{Z v}^{T}=\frac{-\pi \alpha^{2}}{32 \sin ^{4} \theta_{W}} \frac{\beta}{\hat{s}}\left[\left(1-4 \sin ^{2} \theta_{W}\right)\left[\frac{4}{3}\left(3-\beta^{2}\right)+\left(1-\beta^{4}\right)+\frac{\left(1-\beta^{2}\right)}{2 \beta}\left[3-\beta^{2}\left(2+\beta^{2}\right)\right] \mathscr{L}\right]\right. \\
& \left.\left.\quad+\frac{8}{3} \beta^{2}+\left(1-\beta^{4}\right)-\frac{\left(1-\beta^{2}\right)^{3}}{2 \beta}\right] \mathscr{L}\right] .
\end{aligned}
$$

Whereas for the $W$ 's longitudinally polarized, we have

$$
\widehat{\sigma}\left(W_{L}^{+} W_{L}^{-} \rightarrow L^{+} L^{-}\right)=\sum_{i, j} \hat{\sigma}_{i j}^{L} \text {, }
$$

where

$$
\begin{aligned}
& \hat{\sigma}_{A A}^{L}=\frac{\pi \alpha^{2}}{6} \frac{\hat{s}}{M_{W}{ }^{4}} \beta\left(3-\beta^{2}\right), \\
& \hat{\sigma}_{Z Z}^{L}=\frac{\pi \alpha^{2}}{96 \sin ^{4} \theta_{W}} \frac{\hat{s}}{M_{W}^{4}} \beta\left[\left(1-4 \sin ^{2} \theta_{W}\right)^{2}\left(3-\beta^{2}\right)+2 \beta^{2}\right] \text {, } \\
& \hat{\sigma}_{H H}^{L}=\frac{\pi \alpha^{2}}{8 \sin ^{4} \theta_{W}} \frac{M_{L}{ }^{2}}{M_{W}{ }^{4}} \chi_{H} \hat{s}^{2} \beta^{3}, \\
& \hat{\sigma}_{v v}=\frac{\pi \alpha^{2}}{64 \sin ^{4} \theta_{W}} \frac{\hat{s}}{M_{W}{ }^{4}} \beta\left[-\frac{4}{3}\left(6+\beta^{2}\right)+3\left(1+\beta^{2}\right)\left(3-\beta^{2}\right)-\left(1-\beta^{2}\right)^{2}+2 \beta\left(1-\beta^{2}\right)^{2} \mathscr{L}\right], \\
& \hat{\sigma}_{A Z}^{L}=\frac{\pi \alpha^{2}}{12 \sin ^{2} \theta_{W}}\left(1-4 \sin ^{2} \theta_{W}\right) \frac{\hat{s}}{M_{W}^{4}} \beta\left(3-\beta^{2}\right) \text {, } \\
& \hat{\sigma}_{H v}^{L}=\frac{\pi \alpha^{2}}{8 \sin ^{4} \theta_{W}} \frac{M_{L}^{2}}{M_{W}{ }^{4}} \chi_{H} \hat{s}\left(\hat{s}-M_{H}^{2}\right) \beta\left(-\left(1+\beta^{2}\right)+\frac{\left(1-\beta^{2}\right)^{2}}{2 \beta} \mathscr{L}\right) \text {, } \\
& \hat{\sigma}_{A v}^{L}=\frac{\pi \alpha^{2}}{8 \sin ^{2} \theta_{W}} \frac{\hat{s}}{M_{W}{ }^{4}} \beta\left[-\frac{4}{3} \beta^{2}-\frac{\left(1-\beta^{4}\right)}{2}+\frac{\left(1-\beta^{2}\right)^{3}}{4 \beta} \mathscr{L}\right] \text {, } \\
& \hat{\sigma}_{Z_{\nu}}^{L}=\frac{\pi \alpha^{2}}{16 \sin ^{4} \theta_{W}}\left(1-2 \sin ^{2} \theta_{W}\right) \frac{\hat{s}}{M_{W}^{4}} \beta\left[-\frac{4}{3} \beta^{2}-\frac{\left(1-\beta^{4}\right)}{2}+\frac{\left(1-\beta^{2}\right)^{3}}{4 \beta} \mathscr{L}\right) \text {. }
\end{aligned}
$$

There are terms in (15) which exhibit a growing with $\hat{s}$ violating unitarity bounds. As expected, they cancel among themselves when we take the sum over these contributions in (14).

\section{RESULTS AND CONCLUSIONS}

The various contributions due to vector-boson fusion to the rapidity distribution at $y=0$ can be inferred from (1)-(4) and from the cross sections of each subprocess computed in the previous section. In the numerical calculations we have used $M_{W}=82.5 \mathrm{GeV}$ and $M_{Z}=93.8 \mathrm{GeV}$ for the masses of the intermediate vector bosons. For the weak mixing angle we have assumed $\sin ^{2} \theta_{W}=0.226$. As for the Higgs-boson mass we have taken two different sets of data, fixing the Higgs-boson mass at twice and eight times the mass of $Z^{0}$. For the $Q^{2}$-dependent quark distribution functions we have taken the set 2 given in the Appendix of Ref. 3. For the photon distribution in a quark we have used the Weizsäcker-Williams approximation, which yields

$$
\gamma_{q}(x)=\frac{Q^{2} \alpha}{2 \pi} \frac{\left[x^{2}+2(1-x)\right]}{x} \ln \left(\frac{E^{2}}{m_{q}{ }^{2}}\right),
$$




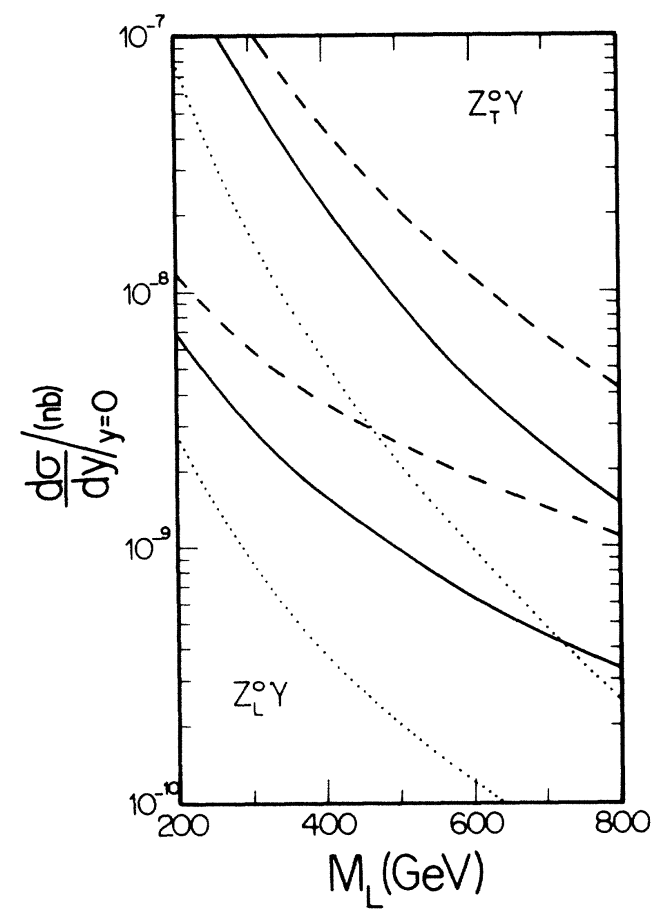

FIG. 3. Rapidity distribution at $y=0$ for $Z^{0} \gamma$ contribution to heavy-lepton pair production in $p p$ collisions for the massive vector boson transversely polarized (upper curves) and longitudinally polarized (lower curves) at $\sqrt{s}=20 \mathrm{TeV}$ (dotted curves), $40 \mathrm{TeV}$ (solid curves), and $70 \mathrm{TeV}$ (dashed curves).

where $m_{q}$ is the quark mass. Our numerical results are summarized in Figs. 3-10. Figure 3 shows the contribution of the subprocess 2(a) to heavy-lepton pair production in $p p$ collisions at different collider energies. In this case we would like to stress the dominance of the transversely polarized $Z^{0}$ boson cross section over the longitudinally polarized one. We credit this dominance to helicity suppression. These cross sections, however, do not give any significant contribution to heavy-lepton pair production, since they are three orders of magnitude smaller than the one associated to the Drell-Yan mechanism ( $q \bar{q}$ fusion).

In Figs. 4 and 5, we show the rapidity distribution at $y=0$ due to $Z^{0} Z^{0}$ fusion for different values of the Higgs-boson mass. We note that the transverse contribution is smaller than the longitudinal one and is more sensitive to the lepton mass. The $Z_{T}^{0} Z_{T}^{0}$ contribution behaves similarly to the two-photon process but, due to the large $Z^{0}$ mass, it lies one order of magnitude below the twophoton contribution ( $\gamma \gamma$ fusion). It is interesting to notice in Fig. 5 the existence of bumps centered at $\boldsymbol{M}_{L}{ }^{2}=\frac{1}{10} \boldsymbol{M}_{H}{ }^{2}$, which is the value that maximizes the width of the Higgs boson in the lepton pair channel.

Figure 6 shows the contribution to heavy-lepton pair production due to the fusion of longitudinally and transversely polarized $W^{+} W^{-}$bosons [Fig. 2(c)] in protonproton collision for $M_{H}=2 M_{Z}$. The transverse contribution is roughly independent of the Higgs-boson mass and, for this reason, we draw in Fig. 7 only the longitudinal contribution for $M_{H}=8 M_{Z}$.

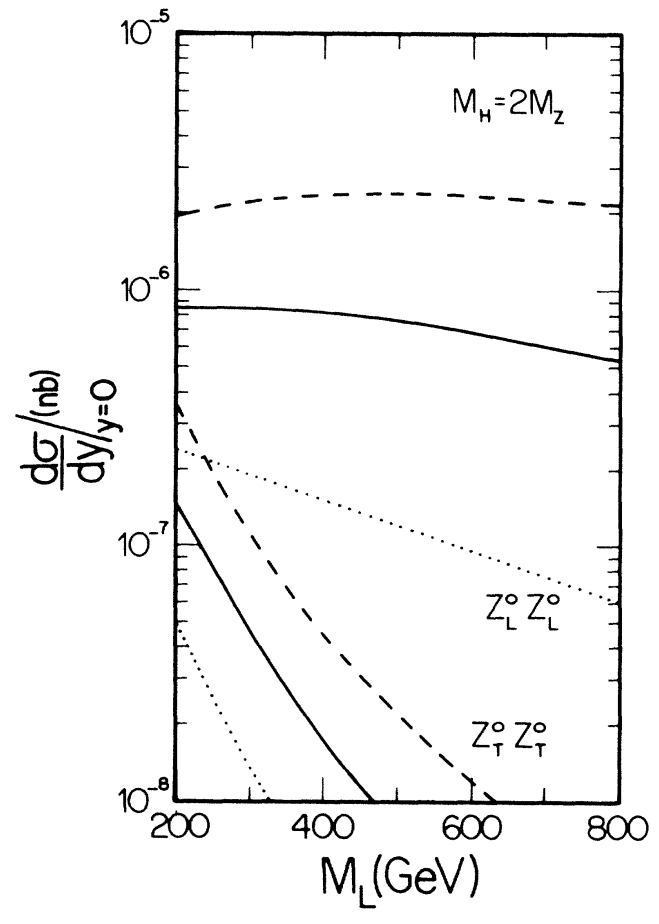

FIG. 4. Rapidity distribution at $y=0$ for $Z^{0} Z^{0}$ contribution to heavy-lepton pair production in $p p$ collisions for both vector bosons longitudinally polarized (upper curves) and transversely polarized (lower curves) for $M_{H}=2 M_{Z}$. The energies are the same as for Fig. 3.

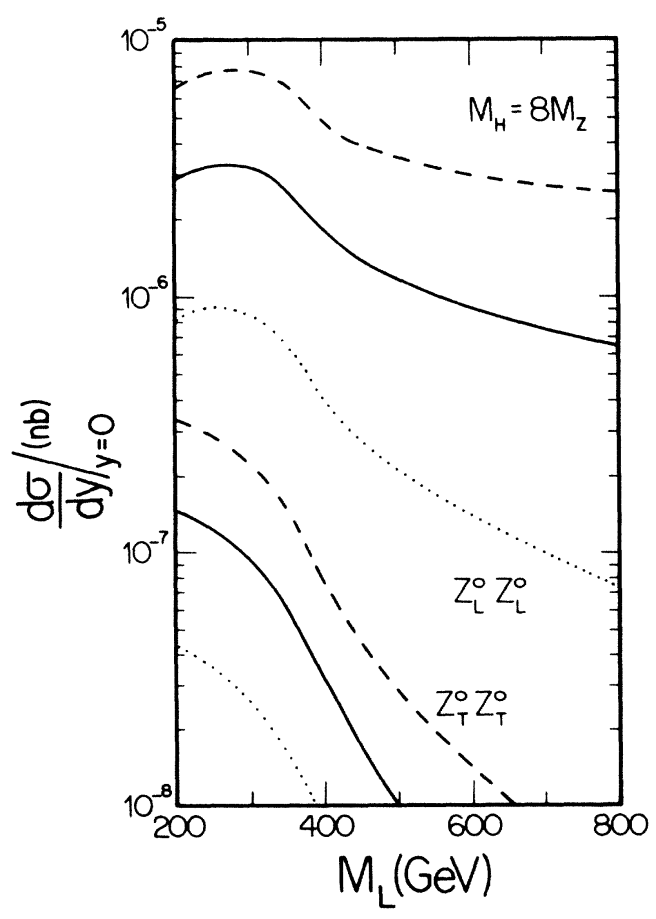

FIG. 5. The same as Fig. 4 for $M_{H}=8 M_{Z}$. 


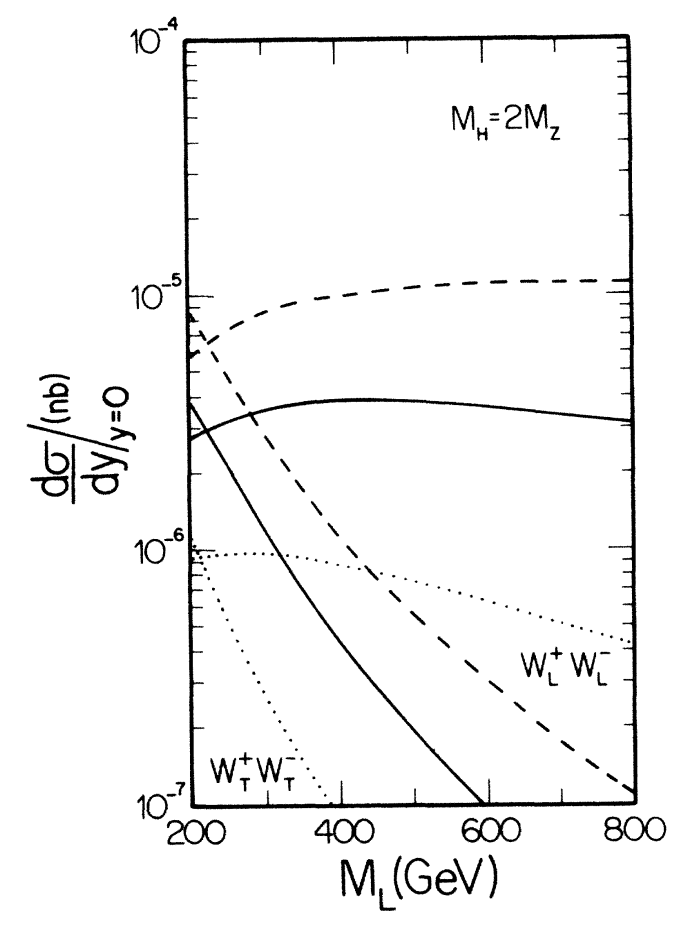

FIG. 6. Rapidity distribution at $y=0$ for $W^{+} W^{-}$contribution to heavy-lepton pair production in $p p$ collisions for both vector bosons longitudinally polarized (upper curves) and transversely polarized (lower curves) for $M_{H}=2 M_{Z}$. The energies are the same as for Fig. 3.

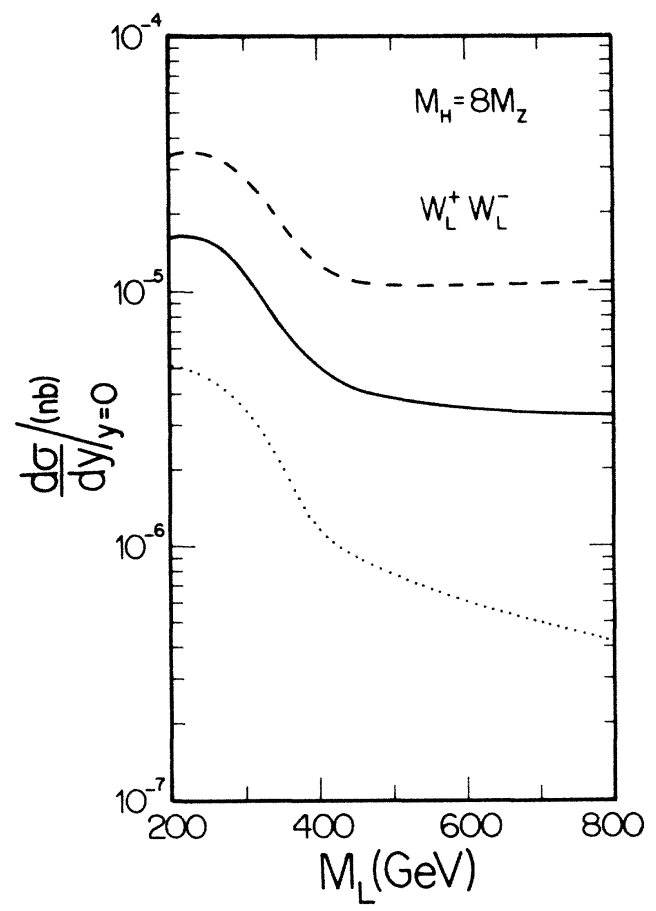

FIG. 7. Rapidity distribution at $y=0$ for $W^{+} W^{-}$contribution to heavy-lepton pair production in $p p$ collisions for both vector bosons longitudinally polarized and for $M_{H}=8 M_{Z}$. The energies are the same as for Fig. 3.

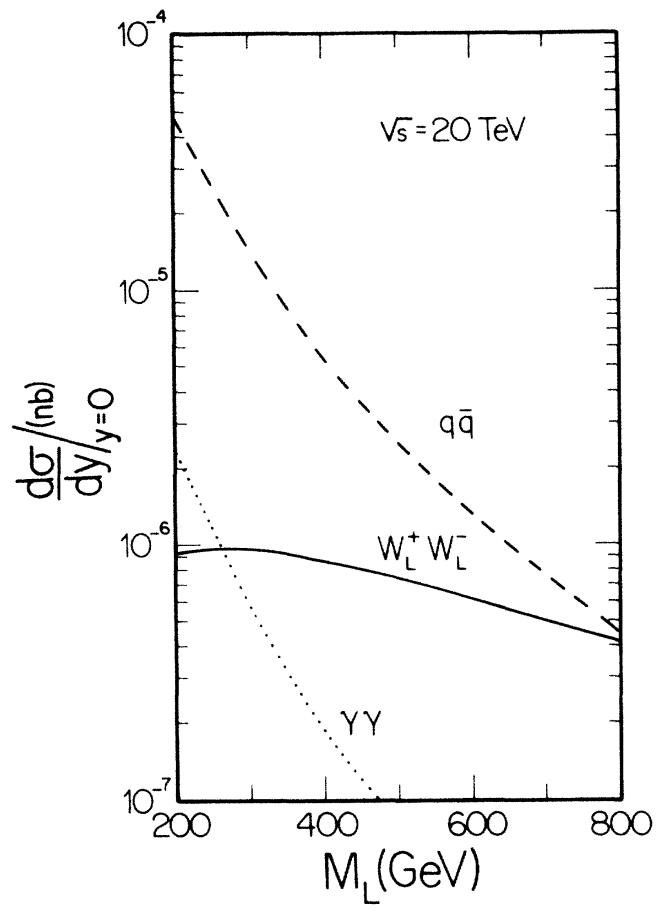

FIG. 8. Rapidity distribution at $y=0$ for Drell-Yan $(q \bar{q})$ (dashed curve), two-gamma fusion $(\gamma \gamma)$ (dotted curve), and two-longitudinally-polarized $W$-boson fusion (solid curve) $\left(M_{H}=2 M_{Z}\right)$ contributions to heavy-lepton pair production in $p p$ collisions at $\sqrt{s}=20 \mathrm{TeV}$.

The relevance of the $W_{L}^{+} W_{L}^{-}$fusion can be inferred by analyzing Figs. 8-10 for $\sqrt{s}=20,40$, and $70 \mathrm{TeV}$. We have also exhibited, for the sake of completeness, the contribution due to $q \bar{q}$ fusion (Drell-Yan mechanism) and $\gamma \gamma$ fusion. From these results it can be inferred that although the Drell-Yan mechanism is the most relevant one for "light leptons," the longitudinal $W$-boson fusion dominates over the Drell-Yan mechanism for heavy leptons $\left(M_{L} \gtrsim 600 \mathrm{GeV}\right.$ at $\sqrt{s}=40 \mathrm{TeV}$, whereas $M_{L} \gtrsim 500 \mathrm{GeV}$ at $\sqrt{s}=70 \mathrm{TeV})$.

We have verified that all the above conclusions remain valid when we consider proton-antiproton instead of proton-proton collisions.

It has been pointed out in Ref. 2 that the effective- $W$ approximation, used throughout this paper, is reliable only when the scattering amplitude is dominated by one of the modes (longitudinal or transverse) in a clear way. Hence, for processes satisfying the above criterion, we do not need to worry about the interference between transverse and longitudinal bosons inside a proton. This condition is met for all processes we analyzed, except in the case of the fusion of $W$ 's for lepton masses smaller than $\sim 300 \mathrm{GeV}$. Thus the use of the effective-vector-boson approximation is not reliable in this situation.

The effective-vector-boson approximation provides quite good results. In fact for the production of heavy Higgs bosons, $\mathrm{Cahn}^{4}$ has shown that using the effectivevector-boson approximation, the cross section is $5-30 \%$ 


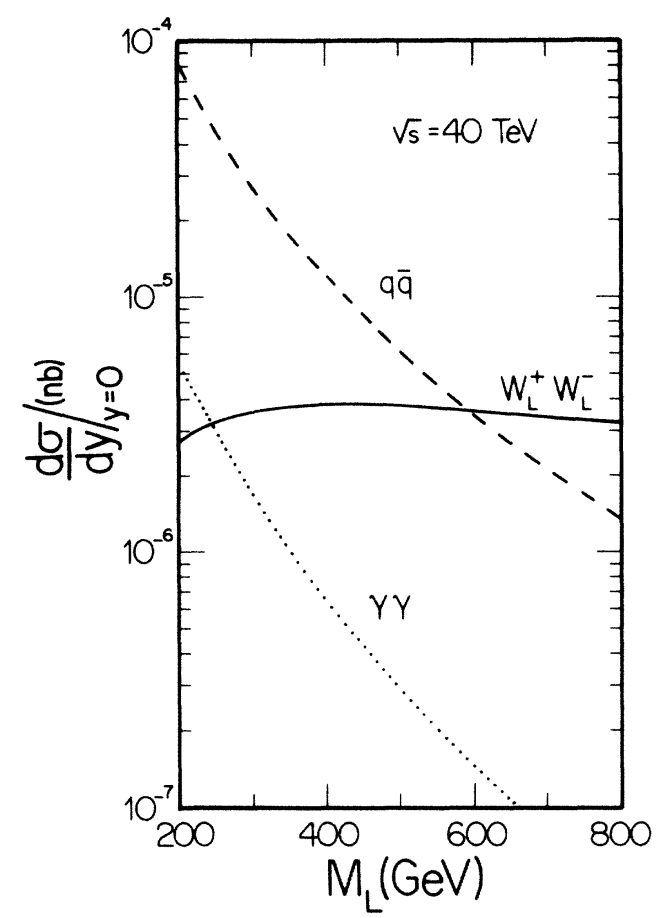

FIG. 9. The same as Fig. 8 at $\sqrt{s}=40 \mathrm{TeV}$.

larger than the correspondent exact result.

Recently, Willenbrock, and Dicus ${ }^{5}$ analyzed the gluonfusion mechanism for heavy-lepton production, which is particularly enhanced if, as they assumed, there is a contribution of a loop of a new heavy quark. Their proposal entails a cross section roughly one order of magnitude larger than the $W_{L}^{+} W_{L}^{-}$fusion mechanism. Nevertheless, we expect that for the production of a heavy lepton $(L)$ and its neutral partner $\left(v_{L}\right)$ the vector-boson fusion studied here will be the most important mechanism since in this case there will not be competition from the gluongluon fusion process. This reaction is now under investigation by us.

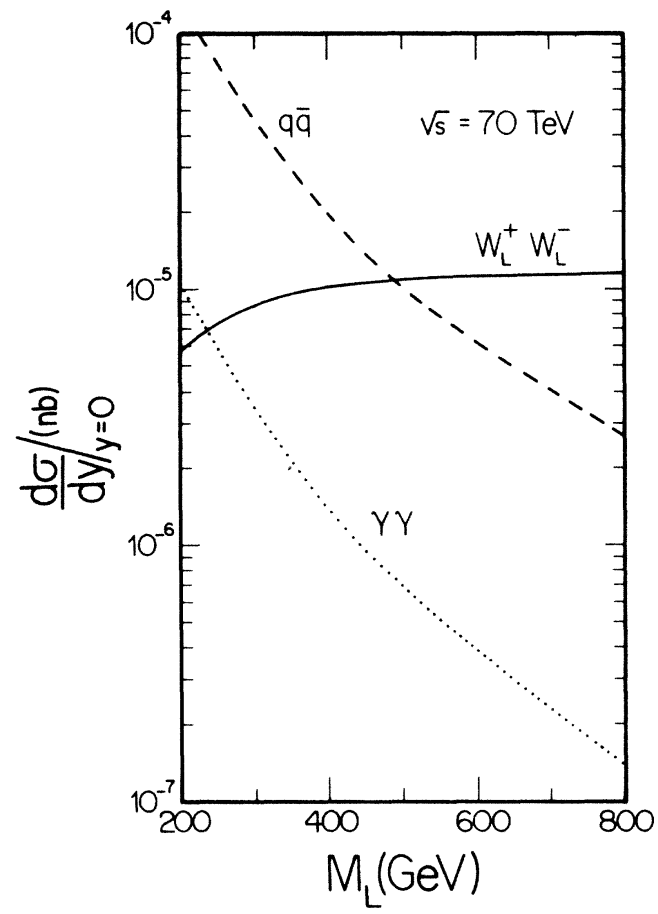

FIG. 10. The same as Fig. 8 at $\sqrt{s}=70 \mathrm{TeV}$.

\section{ACKNOWLEDGMENTS}

We wish to thank R. Aldrovandi and V. Pleitez for enlightening discussions and C. O. Escobar for the critical reading of the manuscript. We are indebted to Fundação de Amparo à Pesquisa do Estado de São Paulo (S.F.N.), Conselho Nacional de Desenvolvimento Científico e Tecnológico, Brasil and the Financiadora de Estudos e Projetos (G.C.M. and A.A.N.) for their financial support. We are grateful to the Instituto de Física Teórica (S.F.N.) and Departamento de Física Matemática da USP (O.J.P.E.) for their kind hospitality.
${ }^{1}$ G. L. Kane, W. W. Repko, and W. B. Rolnick, Phys. Lett. 148B, 367 (1984).

${ }^{2}$ Sally Dawson, Nucl. Phys. B249, 42 (1985).

${ }^{3}$ E. Eichten, I. Hinchliffe, K. Lane, and C. Quigg, Rev. Mod.
Phys. 56, 579 (1984).

${ }^{4}$ R. N. Cahn, Nucl. Phys. B255, 341 (1985).

${ }^{5}$ S. S. D. Willenbrock and D. A. Dicus, Phys. Lett. 156B, 429 (1985). 